\title{
Comparison of Different Models for Residuary Resistance Prediction
}

\author{
Elizabeta Lazarevska \\ Faculty of Electrical Engineering and Information Technologies - Skopje, University Ss. Cyril and Methodius - Skopje, \\ Macedonia, elizabeta. lazarevska@feit.ukim.edu.mk
}

\begin{abstract}
The paper presents several unconventional models of residuary resistance based on fuzzy logic and neural network techniques. First, two fuzzy models are built based on different hull parameters and different Froude numbers. These models are identified by a modification of Sugeno and Yasukawa identification algorithm. Next, a neuro-fuzzy model of residuary resistance is build, based on statistical learning theory. The model presents a fuzzy inference system of Takagi and Sugeno type that uses an extended relevance vector machine for learning its parameters and number of fuzzy rules. Finally, a neural network approach is applied to build four different models of residuary resistance. Two of the neural models apply classic extreme learning machine, and the other two implement incremental extreme learning machine philosophies. The obtained models are validated for their generalization and approximation performance, and although they all possess excellent approximation capabilities, our neural models based on extreme learning machine have shown the best simulation results.
\end{abstract}

Keywords: residuary resistance, fuzzy modeling, neuro-fuzzy model, extreme learning machine, random nodes

\section{Introduction}

Obtaining a hydrodynamic model of a sailing yacht is an important step in its initial design, because the model can be used for calculation of the most important hydrodynamic forces acting upon the yacht, evaluation of yacht performance and estimation of its required propulsive power. Within these efforts, Delft Ship Hydromechanics Laboratory at Delft University of Technology in Nederland has produced several series of yacht models, known together as Delft Systematic Yacht Hull series (DSYHS). This large data base of sailing yacht models today consists of 7 series with a total of approximately 70 models and can be accessed through DSYHS Data Base. According to (Keuning and Katgert, 2008), it is probably the largest series of yacht hulls systematically designed, built, and tested up to now and the series is still expanding. DSYHS is elaborated in much detail in (Keuning and Sonnenberg,
1998). DSYHS has been used for extensive research of sailing yacht hydrodynamics and performance over the past five decades (Keuning and Katgert, 2008; Keuning and Sonnenberg, 1998; Kerwin, 1978; Gerritsma et al., 1981; Gerritsma and Keuning, 1988; Gerritsma et al., 1992; Keuning et al., 1996; Keuning and Binkhorst, 1997). The research presented in this work is based on DSYHS also and deals with prediction of residuary resistance in sailing yachts.

The prediction of total yacht resistance, and particularly its residuary resistance, is very important because of its influence on ship hull design. This prediction should be done with the highest possible accuracy to ensure that the ship operates at optimal speed under most efficient and cost-effective conditions. There are several models for residuary resistance prediction presented in the literature and obtained through regression analysis (Keuning and Katgert, 2008). The variables in these models are different parameters describing hull geometry, and the models are given as sets of polynomials of rather complex structure. Their coefficients are valid only for a specific ship speed, described by a corresponding Froude number. Thus, large look-up tables must be built for each model for different discrete values of Froude number.

This paper proposes and describes several unconventional models for residuary resistance prediction in sailing yachts. The modeling is done on the Yacht Hydrodynamic Data Set available at (Lichman, 2013). The set includes 308 full-scale experiments performed at Delft Ship Hydromechanics Laboratory, which consist of 22 different hull forms. The supplied input parameters are different coefficients concerning the hull geometry and yacht speed: longitudinal position of the center of buoyancy, prismatic coefficient, length to displacement ratio, beam to draught ratio, length to beam ratio, Froude number. The measured variable, i.e. the output, is the residuary resistance per unit weight of displacement.

\section{Fuzzy Models of Residuary Resistance}

The paper presents two fuzzy models of residuary resistance: a position type and a position - gradient type fuzzy model. The identification of these models is based 
on (Sugeno and Yasukawa, 1993). The obtained position type fuzzy model of residuary resistance is of the following form:

$$
\begin{gathered}
R^{i}: \text { IF } x_{3} \text { is } A_{3}^{i} \text { and } x_{5} \text { is } A_{5}^{i} \text { THEN } y \text { is } B^{i} ; \\
i=1,2, \cdots, 5
\end{gathered}
$$

and is shown in Figure 1. It has two inputs $x_{3}$ and $x_{5}$, one output $y$ and five fuzzy rules $R^{i}(i=1,2, \cdots, c=5)$; $A_{3}^{i}, A_{5}^{i}, B^{i}$ are fuzzy variables with trapezoidal membership functions, and $c$ is the number of clusters. A deffuzification method known as center of gravity is used to infer the model output $\hat{y}$, and $\hat{y}$ is calculated as the weighted average of the centers of gravity $b^{i}$ for the consequent membership functions $\mu_{B^{i}}(y)$, with respect to the weighting factors $w^{i}$ :

$$
\begin{gathered}
\hat{y}=\sum_{i=1}^{c} w^{i} b^{i} / \sum_{i=1}^{c} w^{i} \\
w^{i}=\min _{1 \leq j \leq n} \mu_{A_{j}^{i}}\left(x_{j}\right) ; 1 \leq i \leq c \\
b^{i}=\frac{\int y \mu_{B^{i}}(y) d y}{\int \mu_{B^{i}}(y) d y}
\end{gathered}
$$

The output of the position type fuzzy model of residuary resistance compared to the actual output is shown in Figure 2. The model performance has been evaluated through the performance index PI, defined as root mean square error (RMSE) of the model output:

$$
P I=\sqrt{\frac{1}{N} \sum_{i=1}^{N}\left(y_{\text {real }}^{i}-y_{\text {model }}^{i}\right)^{2}}
$$

The obtained position-gradient fuzzy model of residuary resistance is shown in Figure 3 and is of the following form:
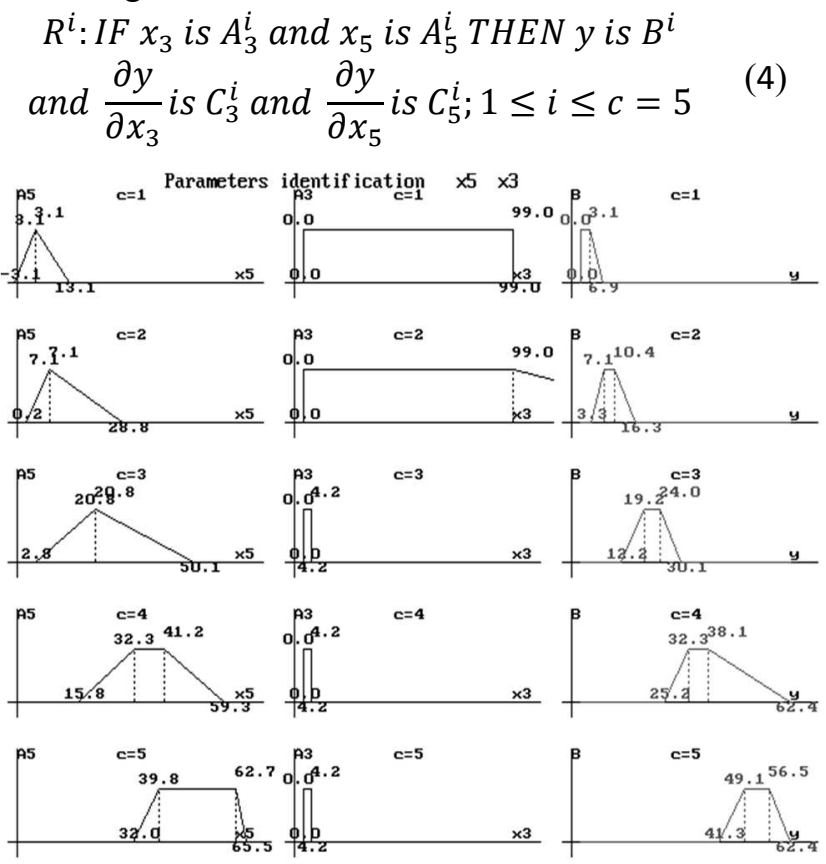

Figure 1. A position type fuzzy model of residuary resistance.

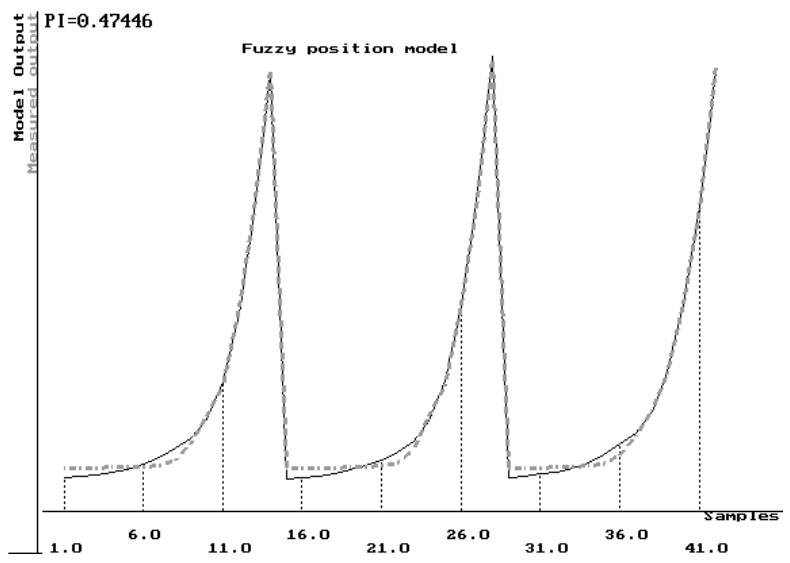

Figure 2. The output of the position type fuzzy model of residuary resistance (dashed line) compared to the actual measured output (solid line).
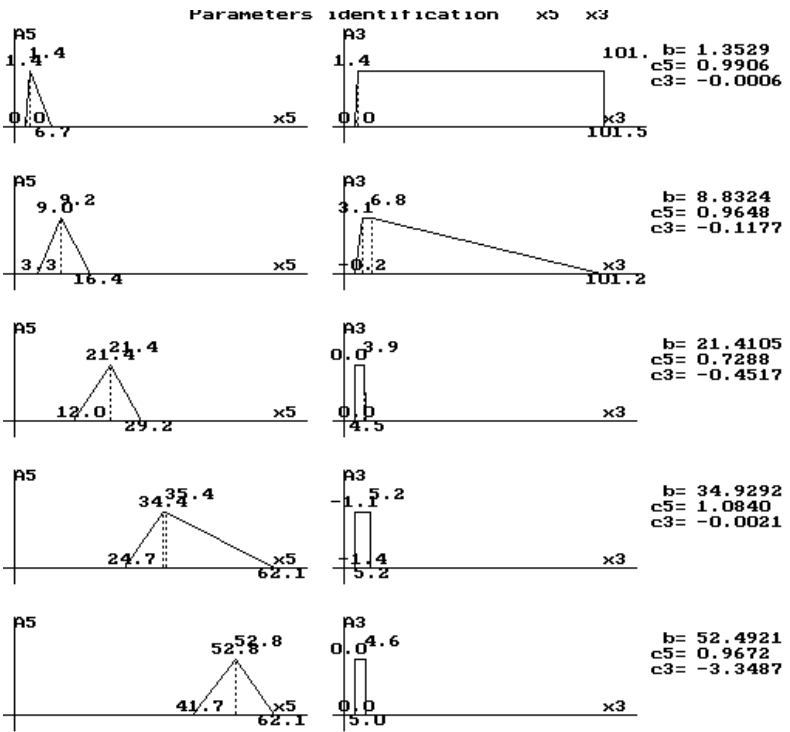

Figure 3. A position-gradient type fuzzy model of residuary resistance.

where $A_{3}^{i}, A_{5}^{i}, B^{i}, C_{3}^{i}, C_{5}^{i}$ are fuzzy variables with trapezoidal membership functions and $\partial y / \partial x_{3}, \partial y / \partial x_{5}$ are partial derivatives of the fuzzy rule outputs with respect to the corresponding inputs. The difference between the two fuzzy models is since a position type fuzzy model cannot be built over the whole input space when some data are missing. In these cases, the output of the model can be estimated through extrapolation based on the local fuzzy rules, which leads to a positiongradient fuzzy model. The output of this model is inferred in the following way:

$$
\hat{y}=\frac{\sum_{i=1}^{c} w\left(d^{i}\right)\left\{\left[b^{i}+\sum_{j=1}^{n}\left(d_{j}^{i} \times c_{j}^{i}\right)\right]\right\}}{\sum_{i=1}^{c} w\left(d^{i}\right)}
$$

where $b^{i}$ and $c_{j}^{i}$ are values obtained by defuzzification of $B^{i}$ and $C_{j}^{i}$, respectively; $d^{i}$ is the distance between the input and the core region of the $i-$ th fuzzy rule; $d_{j}^{i}$ is a component of $d^{i}$ on the $x_{j}$ coordinate axis and $w\left(d^{i}\right)=$ $\exp \left(-d^{i}\right)$ is the weight of the $i-$ th fuzzy rule with 
respect to distance $d^{i}$. The performance of the positiongradient type fuzzy model is evaluated through its PI based on RMSE criterion (3) and is shown in Figure 4.

The applied identification algorithm (Sugeno and Yasukawa, 1993) is a very well-known method for fuzzy identification. Several modifications of this identification method have been presented in literature (Tikk, 2002; Haddad, 2008; Kim et al., 1997; Lazarevska and Trpovski, 2000). In this research, the modification presented in (Lazarevska and Trpovski, 2000 ) is used. The performed algorithm includes parameter identification at each stage of model's rule structure identification process, thus significantly improving the accuracy of the obtained intermediate fuzzy models. As a result, a more efficient and more accurate selection of inputs to the identified fuzzy models is obtained. The parameter identification is done both for the premise and the consequent parameters of the fuzzy rules. In addition, parameter identification is done throughout the process of estimation of partial derivatives of the output with respect to the input variables. As a result, the two obtained fuzzy models very successfully model the given input output data, generating the desired output only by two significant inputs, opposite conventional polynomial models that struggle with many parameters and parameter dependent coefficients. The identification of these models in much more detail is given in (E. Lazarevska, 2016).

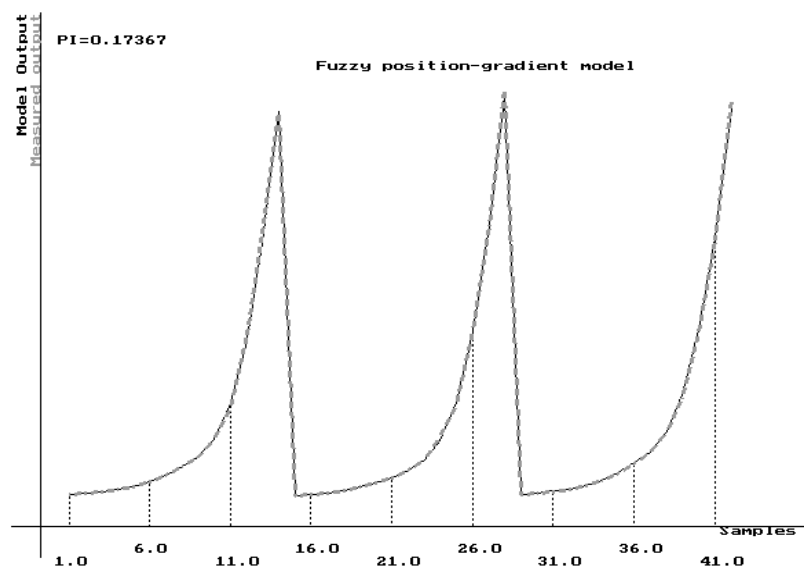

Figure 4. The output of the position-gradient type fuzzy model of residuary resistance (dashed line) compared to the actual measured output (solid line).

\section{A Neuro-fuzzy Model for Residuary Resistance Prediction}

The neuro-fuzzy model for residuary resistance prediction presented in this paper is based on several excellent papers (Vapnik, 1998; Tipping, 2001, Kim et al., 2006) and is described in detail in (Lazarevska, 2016). The modeling is done on the available inputoutput data $\left\{x_{k}, y_{k}\right\} ; k=1,2, \cdots, N$ and the model has the structure of a Takagi and Sugeno (TS) fuzzy model:

$$
\begin{gathered}
R^{i}: \text { IF } x_{1} \text { is } A_{1}^{i} \text { and } x_{2} \text { is } A_{2}^{i} \text { and } \cdots \text { and } x_{M} \text { is } \\
A_{M}^{i} \text { THEN } f_{i}=a_{i 1} x_{1}+\cdots+a_{i M} x_{M}+a_{i 0} ; \\
i=1,2, \cdots, n
\end{gathered}
$$

where $x_{j}(j=1,2, \cdots, M)$ are inputs to the fuzzy rules $R^{i}(i=1,2, \cdots, n), A_{j}^{i}$ are appropriate fuzzy sets, $a_{i j}$ are consequent parameters, $f_{i}$ is the $i$-th local output variable, $n$ is the number of fuzzy rules and $M$ is the dimension of the input data vectors. The fuzzy sets $A_{j}^{i}$ are represented by Gaussian type kernel functions:

$$
\begin{gathered}
K\left(x_{j}, x_{i j}^{*}\right)=\exp \left[-\frac{\left(x_{j}-x_{i j}^{*}\right)^{2}}{2 \theta_{i j}^{2}}\right] ; \\
i=1,2, \cdots, n ; j=1,2, \cdots, M
\end{gathered}
$$

where $x_{j}$ is the $j$-th feature of the $k$-thinput variable $x_{k}, x_{i j}^{*}$ is the center and $\theta_{i j}$ is the variance of the Gaussian kernel function $K\left(x_{j}, x_{i j}^{*}\right)$ and $i=1,, \cdots, n ; j=1,2, \cdots, M$. Thus, the fuzzy IF-THEN rules (6) have the following specific form (Kim et al., 2006):

$$
\begin{gathered}
R^{i}: \text { IF } x_{1} \text { is } K\left(x_{1}, x_{i 1}^{*}\right) \text { and } x_{2} \text { is } K\left(x_{2}, x_{i 2}^{*}\right) \text { and } \\
\cdots \text { and } x_{M} \text { is } K\left(x_{M}, x_{i M}^{*}\right) \\
\text { THEN } f_{i}=a_{i 1} x_{1}+\cdots+a_{i M} x_{M}+a_{i 0} \\
i=1,2, \cdots, n
\end{gathered}
$$

The function $K\left(x_{j}, x_{i j}^{*}\right)$ in (8) represents the grade of membership of $x_{j}$ with respect to the fuzzy set $A_{j}^{i}, x_{i j}^{*}$ is a relevance vector (RV), $\theta_{i j}$ is a kernel parameter, the number of fuzzy rules $n$ equals the number of RVs and $i=1,, \cdots, n ; j=1,2, \cdots, M$. The number of fuzzy rules and the parameters of the membership functions in (8) are generated automatically by the extended relevance vector learning machine RVM algorithm (Kim et al., 2006). The identification algorithm performs system optimization and generalization simultaneously. The gradient ascent method adjusts the parameters of the kernel functions. The coefficients in the consequent part of the fuzzy rules are determined by the least square method.

The structure of the neuro-fuzzy model of residuary resistance is shown in Figure 5. It is presented as a neural network with six different layers. The first layer is the input layer. It has a total of $M$ nodes, $M$ being the number of elements in the training input vector $\mathbf{x}_{\mathrm{k}}=$ $\left(x_{k 1}, x_{k 2}, \cdots, x_{k M}\right)$. This layer transmits the upcoming input data to the second layer and does not perform any operations over the training input data. The second layer is a fuzzification layer. Each node in this layer has exactly $M$ inputs. The second layer consists of $n$ nodes that represent adequate kernel functions $K\left(x_{j}, x_{i j}^{*}\right)$. From the fuzzy modeling perspective, the Gaussian kernel function is the membership function $K\left(x_{j}, x_{i j}^{*}\right)=$ $\mu_{A_{j}^{i}}\left(x_{j}\right)$ of the $j$-th fuzzy input $x_{j}$ with respect to the $i$ - th fuzzy rule, its parameters $x_{i j}^{*}$ and $\theta_{i j}$ are premise 


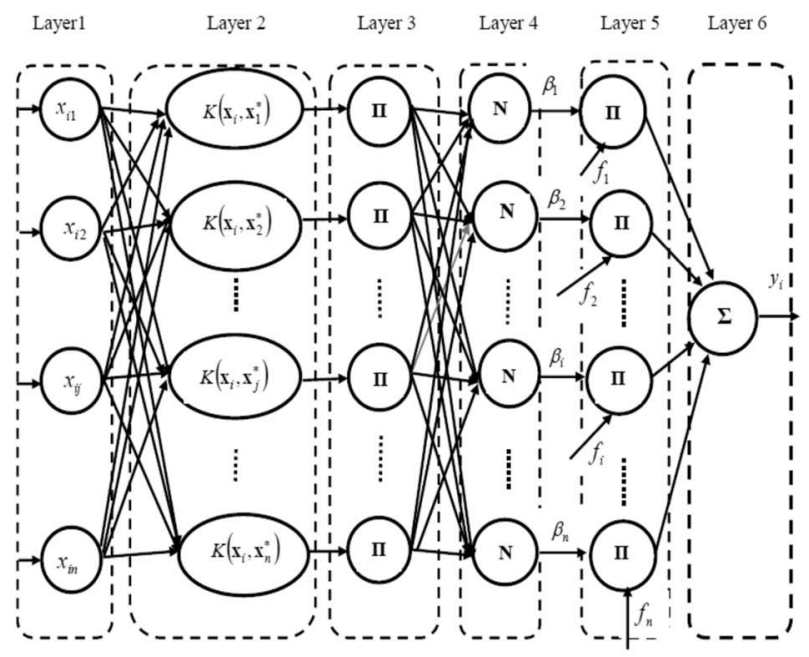

Figure 5. The structure of the neuro-fuzzy model of residuary resistance.

parameters of the corresponding fuzzy rule, $M$ is the number of fuzzy inputs to the neuro-fuzzy model, and the number $n$ of kernel functions is the number of fuzzy rules, i.e. the number of nodes in the second layer. Because of the Gaussian shape of the selected kernel functions, the membership functions of the antecedent part of the fuzzy rules are Gaussian membership functions. From RVM prospective, the center $x_{i j}^{*}$ of the Kernel function is a relevance vector $\mathrm{RV}$, the variance $\theta_{i j}$ is a kernel parameter, and $n$ is the number of RVs. The third layer can be called as the rule layer, since a node in this layer generates the IF part of each fuzzy rule. This layer has $n$ nodes, one for each fuzzy rule, and they compute the firing strength of the associated fuzzy rules using the product of membership functions as T-norm operator. The fourth layer is a normalization layer. It consists of $n$ nodes and each node perform normalization of the firing strength of the associated fuzzy rule. This normalization is done with respect to the sum of the firing strengths of all the fuzzy rules, and the output of each node is the weight $\beta_{i}$ of the corresponding fuzzy rule. Each node $i$ in the fifth layer

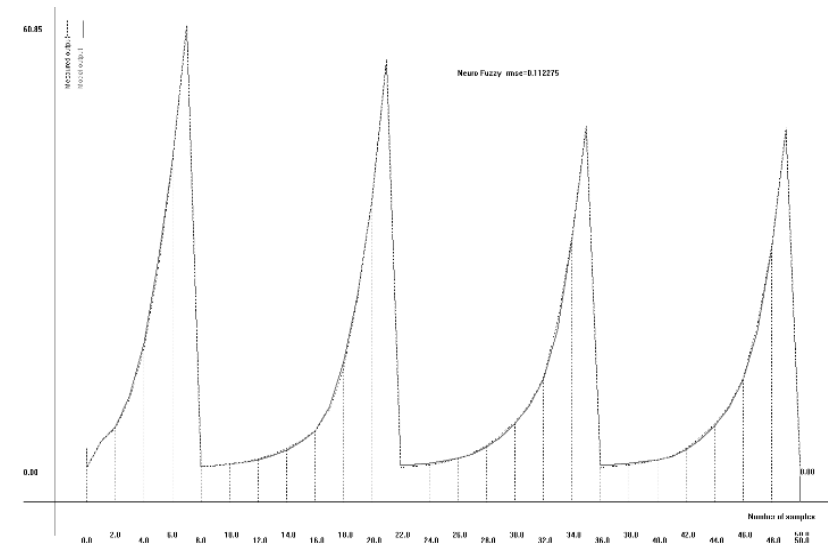

Figure 6. The output of the neuro-fuzzy model of residuary resistance (dashed line) compared to the actual measured output (solid line). calculates the product of the normalized weight $\beta_{i}$ for the $i$-th rule and the local output variable $f_{i}$ of the fuzzy model. The sixth and the last layer is the output layer. The single node in this layer computes the overall output $f(\mathbf{x})$ of the neuro-fuzzy model as the sum of all incoming signals $\beta_{i} f_{i}(i=1,2, \cdots, n)$. The output of the neuro-fuzzy model for residuary resistance prediction with the obtained relevance vectors, and compared to the actual measured output, is shown in Figure 6.

\section{Neural Models of Residuary Resistance based on ELM}

Three neural modes of residuary resistance are presented in this section, based on classic and incremental extreme learning machine (ELM). First the classic ELM is used to obtain the desired model of residuary resistance. To perform the modeling, the available experimental data were divided into two sets: training data and testing data, and fixed number of hidden nodes $n=25$ were assigned for training. Since the approximation performance of classic ELM is generally independent of the type of activation function (Huang and Babri, 1998), the logistic function was chosen for the hidden neurons, and the input parameters of the hidden neurons $\mathbf{w}_{\mathbf{i}}$ and $b_{i}(i=1, \cdots, n)$ were randomly assigned according to the uniform probability distribution. The neural model of residuary resistance based on the classic ELM is of the following form (Huang et al., 2004):

$$
\begin{gathered}
\tilde{y}_{k}=\sum_{i=1}^{n} \mathbf{v}_{i} g_{i}\left(\mathbf{x}_{k}\right)=\sum_{\substack{i=1 \\
k}}^{n} \mathbf{v}_{i} g\left(\mathbf{w}_{i} \mathbf{x}_{k}+b_{i}\right) ; \\
k, \cdots, N
\end{gathered}
$$

where $\mathbf{x}_{\mathrm{k}}=\left[\begin{array}{llll}x_{k 1} & x_{k 2} & \cdots & x_{k M}\end{array}\right]^{T}$ is the $k-$ th input vector of dimension $M, \mathbf{w}_{\mathrm{i}}=\left[\begin{array}{llll}w_{i 1} & w_{i 2} & \cdots & w_{i M}\end{array}\right]^{T}$ are the weights of the connections between the $M$ input neurons and the $i$ - th hidden neuron, $\mathbf{v}_{\mathbf{i}}=\left[\begin{array}{llll}v_{i 1} & v_{i 2} & \cdots & v_{i L}\end{array}\right]^{T}$ is the vector of the weights defining the connections between the $i-$ th hidden neuron and the $L$ output neurons, $b_{i}$ is the threshold, i.e. the bias of the $i-$ th hidden neuron, and $g_{i}\left(\mathbf{x}_{k}\right)$ is the activation function of the $i-$ th hidden neuron; the term $\mathbf{w}_{i} \mathbf{x}_{k}$ denotes inner product between $\mathbf{w}_{i}$ and $\mathbf{x}_{k}$. The hidden neuron output weights can be determined simply and analytically with an adequate least square method yielding the smallest norm least square solution (Huang et al., 2004):

$$
\mathbf{V}=\mathbf{W}^{+} \mathbf{Y}
$$

where $\mathbf{V}$ is the model adjustable parameter vector, $\mathbf{Y}$ is the model output vector, and $\mathbf{W}^{+}$stands for the Moore-Penrose generalized inverse of the hidden layer output matrix W (Rao and Mitra, 1971). However, the conducted research has shown that the approximation performance of classic ELM depends considerably on the values of the arbitrary chosen weights and biases for the hidden layer inputs. Therefore, a simple approach is used in this research to overcome the uncertainty problem with 
classic ELM. After the selection of appropriate activation function and the number of hidden neurons, the desired value of the error is set as $\varepsilon$. Then the training of the constructed neural network with ELM is conducted and the error of the obtained model is compared to the preset desired value $\varepsilon$. If the model error is greater than $\varepsilon$, the training process is repeated. Otherwise, it is considered that the obtained model has the desired accuracy. Figure 7 shows the output of the ELM model of residuary resistance obtained in this way compared to the actual output.

The approximation accuracy of ELM certainly depends on the number of hidden layer neurons $n$, and when $n$ approaches the number of training samples $N$, the model error approaches zero (Huang and Babri, 1998). However, since too large number of hidden neurons is not a desired neural network feature, (Huang et al., 2006) has proposed a new learning algorithm called as incremental ELM (IELM). The difference between the classic ELM and the IELM is in the addition of new neurons to the hidden layer of the later. The new neurons can be added one at a time, or in groups, and the process of learning continues until the preset maximum number of hidden neurons is reached, or the preset acceptable model error is achieved. As with classic ELM, the input parameters of the hidden layer in IELM are randomly generated and are not adjusted at all during the learning process. When a new hidden neuron is added to the hidden layer, the IELM algorithm does not recalculate the hidden layer output parameters of the existing hidden nodes, and the output weights of the hidden layer are calculated according to (Huang et al., 2006):

$$
\boldsymbol{\beta}_{n}=\mathbf{E} \mathbf{H}^{\mathbf{T}} / \mathbf{H H}^{\mathbf{T}}=\sum_{i=1}^{N} e(i) h(i) / \sum_{i=1}^{N} h^{2}(i)
$$

where $h(i)$ denotes the activation of the added new hidden node for the $i$ - th training sample, while $e(i)$ is the corresponding residual error before the addition of the new hidden node in question; $\mathbf{H}=\left[\begin{array}{lll}h(1) & \cdots & h(N)\end{array}\right]^{\mathrm{T}}$ is the activation vector of the newly added node for all the training samples, and $\mathbf{E}=[e(1) \cdots e(N)]^{T}$ is the vector of residual error before the addition of the new

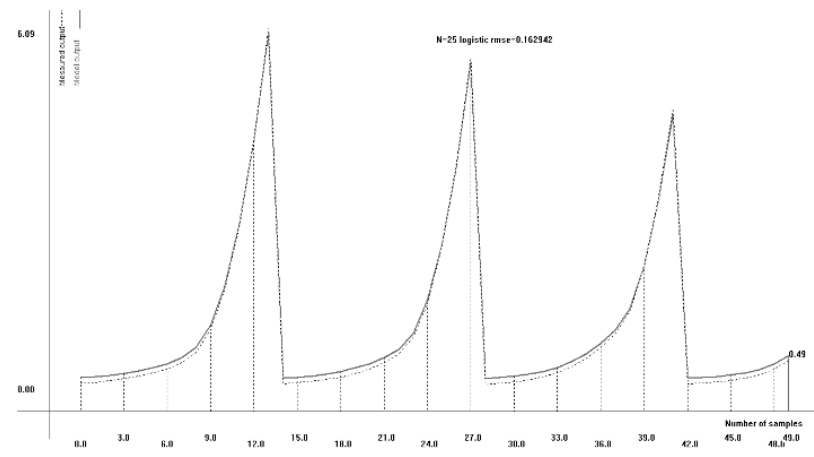

Figure 7. NN model for residuary resistance prediction based on our modification of classic ELM with $\boldsymbol{n}=$ 25, logistic activation function and $\mathrm{RMSE}=0.162942$. hidden neuron. The value of the residual error after the addition of a new hidden neuron is calculated according to (Huang et al., 2006):

$$
\mathbf{E}=\mathbf{E}-\boldsymbol{\beta}_{\mathbf{n}} \mathbf{H}_{\mathbf{n}}
$$

However, the obtained IELM model does not provide the best possible solution considering the model approximation error, since the output weights of the nodes in the hidden layer are not recalculated after each addition of a new hidden node. To overcome this problem, we have tested a much simpler algorithm than the IELM described above, which is a modification of the classic ELM in a sense that it accepts increasing number of hidden nodes and searches for smallest preset error defined by $\varepsilon$. The algorithm recalculates the output parameters of all the hidden neurons in the hidden layer after every new addition to the hidden layer and performs until the preset maximum number of hidden neurons or the preset desired model error is reached. The performance of our version of IELM residuary resistance model with $n=30$ and logistic activation function, compared to the actual measured output, is shown in Figure 8. This model has much better performance index than the IELM given by (11) - (12).

To overcome the accuracy issue with IELM, (Huang and Chen, 2007) proposed a modification of IELM called convex incremental ELM (CIELM), which assigns the output hidden layer weights as:

$$
\begin{aligned}
\boldsymbol{\beta} & =\frac{\mathbf{E}[\mathbf{E}-(\mathbf{Y}-\mathbf{H})]^{\mathbf{T}}}{[\mathbf{E}-(\mathbf{Y}-\mathbf{H})][\mathbf{E}-(\mathbf{Y}-\mathbf{H})]^{T}}= \\
& =\frac{\sum_{i=1}^{N} e(i)\{e(i)-[y(i)-h(i)]\}}{\sum_{i=1}^{N}\{e(i)-[y(i)-h(i)]\}^{2}}
\end{aligned}
$$

The variables in (13) are defined as in (11). The output of the residuary resistance CIELM model according to (13) is given in Figure 9. It has worse PI than the model in Figure 7, which is due to the fixed random assignment of network input parameters.

The performance indices of the obtained models for residuary resistance prediction are shown in Table 1. The model with our version of IELM has shown the best PI value and the reason for this is found in the fact that

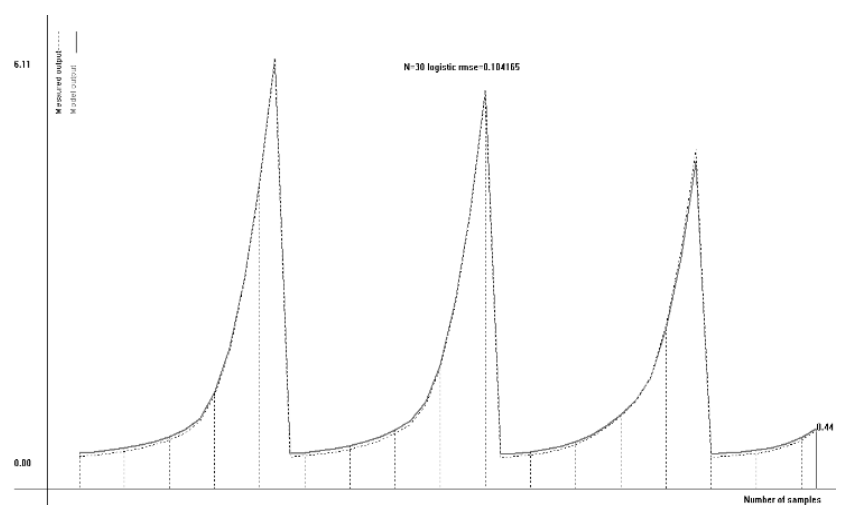

Figure 8. Our version of IELM model for residuary resistance prediction with $\boldsymbol{n}=\mathbf{3 0}$, logistic activation function and $\mathrm{RMSE}=0.104165$. 


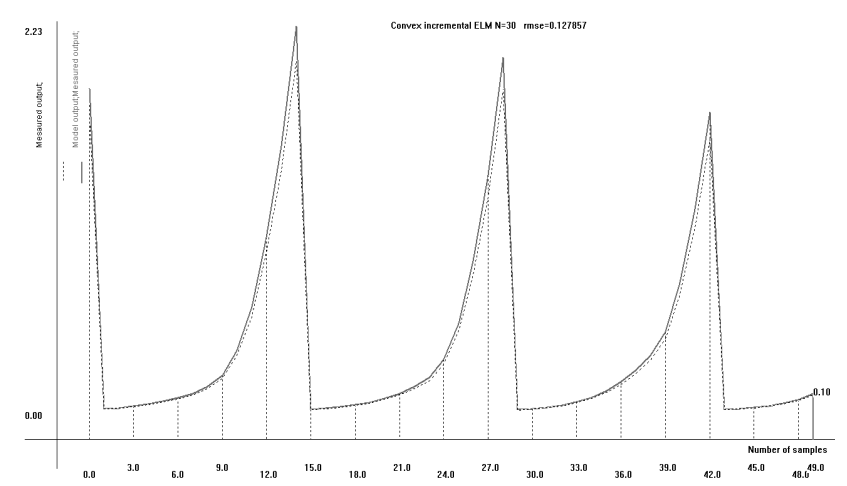

Figure 9. CIELM model for residuary resistance prediction with $\boldsymbol{n}=\mathbf{3 0}$, logistic activation function and $\mathrm{RMSE}=0.127857$ according to (Huang and Chen, 2007).

whenever a new hidden node is added to the network, all the output parameters of the hidden layer are recalculated, and a search is conducted with different random assignments for the network input parameters until the desired error is reached. IELM and CIELM do not take into consideration the influence of the randomness factor. Once assigned, the input parameters of IELM and CIELM networks are never changed.

Table 1. Comparison of Residuary Resistance Prediction Models obtained by Different Modeling Techniques.

\begin{tabular}{|l|l|}
\hline Model & $R M S E$ \\
\hline Position type fuzzy model & 0.47446 \\
\hline Position-gradient type fuzzy model & 0.17367 \\
\hline Neuro-fuzzy model based on RVM & 0.112275 \\
\hline NN model based on our version of ELM & 0.162942 \\
\hline $\begin{array}{l}\text { NN model based on IELM according to } \\
\text { (Huang et al., 2006) }\end{array}$ & 0.143637 \\
\hline $\begin{array}{l}\text { NN model based on our version of } \\
\text { IELM }\end{array}$ & 0.104165 \\
\hline $\begin{array}{l}\text { NN model based on CIELM according } \\
\text { to (Huang and Chen, 2007) }\end{array}$ & 0.127857 \\
\hline
\end{tabular}

\section{Conclusions}

The paper presents six unconventional models for residuary resistance prediction based on fuzzy logic and neural network techniques. All of them possess excellent approximation properties. The applied fuzzy logic approach is especially valuable because of its transparency and ease of interpretability, and because it allows the researcher to determine the most significant input variables that affect the modeled system output and behavior, which is very desirable in cases with large number of input candidates. The position-gradient fuzzy model has a comparable accuracy with the more sophisticated models. The neuro-fuzzy model for residuary resistance prediction has the same excellent approximation property as the rest of the presented models, but it lacks the simplicity and the computational speed of the ELM neural models. The neural models for residuary resistance prediction based on ELM philosophy, have clearly showed that ELM indeed possesses the attributes of extreme simplicity, extremely good approximation performance, and extremely fast computation. Very notably, our version of IELM produces the best approximation performance, meaning the smallest approximation error defined as RMSE, because it takes into consideration the effect of input parameters randomness on the ELM.

\section{References}

DSYHS, DSYHS Data Base. Available at: http://dsyhs.tudelft.nl/dsyhs.php (accessed 06.02.2016)

J. Gerritsma and J. A. Keuning. Performance of light- and heavy-displacement sailing yachts in waves. In The 2nd Tampa Bay Sailing Yacht Symp., St. Petersburg, 1988.

J.Gerritsma, J. A. Keuning and R. Onnink. Sailing yacht performance in calm water and in waves. Report No. 925$\mathrm{P}$, In $12^{\text {th }}$ Int. Symp. on Yacht Design and Construction HISWA, 1992.

J. Gerritsma, R. Onnink and A. Versluis. Geometry, resistance and stability of the Delft systematic yacht hull series. Int. Shipbuilding Progress, 28: 276- 297, 1981.

A. H. Hadad, T. Gedeon, S, Shahbazi and S. Bahrami. A modified version of Sugeno-Yasukawa modeler. In 13th International CSI Computer Conference, CSICC 2008 Kish Island, Iran, March 9-11, 2008, 852-856.

G. -B. Huang, and H. A. Babri. Upper bounds on the number of hidden neurons in feedforward networks with arbitrarily bounded nonlinear activation functions. IEEE Trans. Neural Networks, 9: 224-229, 1998.

G. -B. Huang, and L. Chen. Convex incremental extreme learning machine. Neurocomputing, 70: 3056-3062, 2007.

G. -B. Huang, L. Chen, and C. -K. Siew. Universal approximation using incremental constructive feedforward networks with random hidden nodes. IEEE Trans. Neural Net., 17(4): 879-892, 2006.

G. -B. Huang, Q. -Y. Zhu and C. -K. Siew. Extreme learning machine: A new learning scheme of feedforward neural networks. In Proceedings of the IEEE International Joint Conference on Neural Networks (IJCNN2004), 25-29 July, Budapest, Hungary, 2004, 985-990.

J. E. Kerwin. A velocity prediction program for ocean racing yachts. Report 78-11, Department of Ocean Engineering, MIT, 1978.

J. A. Keuning and B. J. Binkhorst. Appendage resistance of a sailing yacht hull. In 13th Chesapeake Sailing Yacht Symp., 1997.

J. A. Keuning and M. Katgert,. A bare hull resistance prediction method derived from the results of the Delft Systematic Yacht Hull Series extended to higher speeds. In Int. Conf. on Innovation in High Performance Sailing Yachts, France, 2008.

J. A. Keuning, R. Onnink, A. Versluis, and A. Van Gulik. The bare hull resistance of the Delft Systematic Yacht Hull Series. In Int. HISWA Symp. on Yacht Design and Construction, Amsterdam RAI, 1996. 
J. A. Keuning and U. B. Sonnenberg. Approximation of the hydrodynamic forces on a sailing yacht based on the Delft Systematic Yacht Hull Series. In Int. HISWA Symp. on Yacht Design and Construction, Amsterdam RAI, 1998, 99152.

E. Kim, M. Park, S. Ji and M. Park. A new approach to fuzzy modeling. IEEE Transactions on Fuzzy Systems, 5(3): 328337, 1997.

J. Kim, Y. Suga and S. Won. A new approach to fuzzy modeling of nonlinear dynamic systems with noise: relevance vector learning mechanism. IEEE Trans. on Fuzzy Systems, 14: 222-231, 2006.

E. Lazarevska. Fuzzy modeling of residuary resistance in sailing yachts. In XIII International SAUM Conference on Systems, Automatic Control, and Measurements, Niš, Serbia, November 09th-11th, 2016.

E. Lazarevska. A Neuro-Fuzzy Model of the Residuary Resistance of Sailing Yachts. In Proceedings of the IEEE Intelligent Systems IS'2016, Sofia, Bulgaria, 2016, 173-179.

E. Lazarevska and J. Trpovski. A modification of the famous fuzzy model by Sugeno and Yasukawa. In Proceedings of the International Symposium on Applied Automatic Systems $A A S^{\prime 2} 2000$, Ohrid, Macedonia, 2000, 31-35.
M. Lichman. UCI Machine Learning Repository [http://archive.ics.uci.edu/ml]. Irvine, CA: University of California, School of Information and Computer Science. 2013.

C. R. Rao and S. K. Mitra. Generalized Inverse of Matrices and its Applications. Wiley. 1971.

M. Sugeno and T. Yasukawa. A fuzzy-logic-based approach to qualitative modeling. IEEE Trans. on Fuzzy Syst., 1(1): 7-33, 1993.

D. Tikk, G. Biró, L. T. Kóczy, T. D. Gedeon and K. W. Wong. Improvements and critique on Sugeno's and Yasukawa's qualitative modeling. IEEE Transactions on Fuzzy Systems, 10(5): 596-606, 2002.

M. E. Tipping. Sparse Bayesian learning and the relevance vector machine. J. Mach. Learn. Res., 1: 211-244, 2001.

V. N. Vapnik. Statistical Learning Theory. Wiley. 1998. 